\author{
SYLWIA ŁUSZCZYŃSKA \\ Uniwersytet Szczeciński \\ s_kal@o2.pl
}

\title{
Revue des recherches sur l'anxiété langagière relative aux compétences linguistiques et aux étapes du traitement de la langue
}

\author{
Review of research on language anxiety \\ related to language skills and three stages \\ of language learning
}

\begin{abstract}
This article presents the findings of selected empirical research on language anxiety, conducted in various countries and among age groups at diverse proficiency levels, applying different research designs and anxiety measurement instruments. First of all, the role of some language skills in anxiety production is presented. The studies summarized below focus on listening, reading and writing in order to more specifically classify the causes of language anxiety. Then, a discussion of the relationship between anxiety during the three stages of language processing (i.e. input, processing, output) and its relationship to learning deficits is presented.
\end{abstract}

KEYWORDS: language anxiety, students, input, output, processing, performance.

MOTS-CLES: anxiété langagière, étudiants, entrée, sortie, traitement, performance.

\section{ANXIÉTÉ LANGAGIÈRE ET LES COMPÉTENCES LINGUISTIQUES}

Outre le rôle de la compétence orale analysée en référence à l'anxiété langagière et à la réussite dans l'apprentissage d'une langue (cf. article de Kalińska-Łuszczyńska « Revue des recherches empiriques sur l'anxiété langagière et la réussite » qui est en cours d'impression), le rôle des autres 
compétences linguistiques dans la création de l'anxiété mérite une étude approfondie. Les études résumées ci-dessous sont axées sur l'écoute, la lecture et l'écriture pour classifier plus précisément les causes de l'anxiété langagière.

L'objet de la recherche réalisée par Saito et al. (1999) est d'identifier l'anxiété de lecture et d'analyser sa relation avec la spécificité de la langue cible et son niveau de difficulté. Elle porte sur 383 étudiants américains en premier semestre d'un cours universitaire de français (langue « apparentée ») $(\mathrm{N}=192)$, de japonais (langue "non apparentée ») $(\mathrm{N}=114)$ et de russe (langue "semi-apparentée ") ( $\mathrm{N}=77)$. Les techniques utilisées dans l'étude ont été utilisée notamment : le FLRAS (Foreign Language Reading Anxiety Scale), mesurant l'anxiété relative à différents aspects de la lecture et les perceptions de la difficulté de la lecture par rapport à la difficulté des autres compétences linguistiques, et le FLCAS (Foreign Language Classroom Anxiety Scale), mesurant le niveau de l'anxiété langagière générale, avec les notes finales de cours comme mesure globale de la performance des apprenants.

Les principaux résultats montrent que l'anxiété relative à la lecture en langue étrangère se distingue de l'anxiété langagière générale, en dépit de la relation très proche entre ces concepts, mais les niveaux généraux d'anxiété de lecture sont légèrement inférieurs à ceux de l'anxiété langagière. L'anxiété de lecture a une corrélation négative avec la performance des apprenants mesurée par les notes finales. L'anxiété langagière ne dépend pas de la combinaison donnée de la langue maternelle et de la langue cible, alors que l'anxiété de lecture varie en fonction de la langue cible. L'anxiété de lecture est la plus élevée en ce qui concerne la lecture en japonais, une langue non apparentée ayant un système d'écriture inconnu et non latin, tandis que, ce qui est tout à fait surprenant, la lecture en français est plus difficile qu'en russe. Les auteurs attribuent cette constatation au fait que bien que le français utilise l'alphabet latin, les apprenants se rendent compte pendant l'apprentissage que c'est une langue phonétiquement plus complexe que leur langue maternelle. Généralement, l'anxiété de lecture est positivement corrélée avec le niveau de difficulté de la lecture dans une langue étrangère.

Une analyse approfondie de l'anxiété d'écriture et de ses liens avec l'anxiété langagière est le sujet de l'étude de Cheng et al. (1999). 433 étudiants de lettres anglaises d'une université taïwanaise fréquentant un cours de composition et des cours de conversation en anglais ont participé à cette recherche. Le questionnaire, outil principal de recherche, était composé d'une échelle FLCAS modifiée, une version modifiée de la langue seconde du test d'appréhension de lecture de Daly et Miller (1975), appelée Test d'anxiété de l'écriture dans une langue seconde (SLWAT : Second Language Writing Apprehension Test) et les données démographiques sur les partici- 
pants, ainsi que d'autres informations pertinentes (comme la maîtrise autoperçue de l'expression orale et de l'écriture en anglais).

Les résultats de la recherche établissent l'indépendance relative des deux concepts en question : celui de l'anxiété d'apprentissage d'une langue seconde en classe et celui de l'anxiété d'écriture dans une langue seconde, ce qui signifie qu'elles sont liées et caractérisées par une perception négative par le sujet de ses propres compétences linguistiques. Néanmoins, elles constituent des concepts d'anxiété relativement distincts. En outre, la confiance en soi, un sous-composant de l'anxiété d'apprentissage d'une langue seconde en classe plus générale ou de l'anxiété de production écrite dans une langue seconde, s'est avérée être un prédicteur de différents niveaux d'anxiété. Une autre constatation importante concerne la nature des deux concepts d'anxiété analysés. Une association plus forte de l'échelle FLCAS avec la compétence orale conduit à la conclusion que le FLCAS est une mesure de l'anxiété liée à des situations de prise de parole, mais en même temps à l'écriture. C'est donc une mesure des préoccupations générales des apprenants à l'égard des cours de langue seconde avec un élément d'anxiété forte liée à l'expression orale. De plus, l'anxiété d'écriture dans une langue seconde, mesurée par l'échelle SLWAT, est une anxiété de compétence linguistique qui pourrait bien désigner une émotion négative et des attitudes formées pendant le processus d'apprentissage de la langue seconde.

L'étude réalisée par Argaman et Abu-Rabia (2002) vise à analyser la relation entre les niveaux d'anxiété langagière et les tâches de lecture et d'écriture. Ses participants étaient 68 élèves de premier cycle du secondaire dans le nord d'Israël, locuteurs natifs d'hébreu et apprenant l'anglais comme langue étrangère. Les outils utilisés dans l'étude étaient une échelle mesurant l'anxiété langagière, adaptée à partir de l'échelle FLCAS, une tâche de compréhension écrite et une tâche d'écriture.

Les résultats de l'étude montrent des corrélations négatives entre l'anxiété langagière et les résultats des tâches de lecture et d'écriture. On fait valoir que, en dépit de l'accent principal mis sur l'anxiété langagière relative à la communication orale, l'anxiété influence les compétences en langue étrangère dans tous les domaines. En outre, les auteurs attribuent une faible performance relative aux compétences en compréhension à un manque de compétences en langue étrangère.

Le développement d'un outil valide et fiable pour mesurer l'anxiété de production écrite en langue seconde est l'objet principal de la recherche effectuée par Cheng (2004). La technique la plus couramment utilisée est le test d'appréhension d'écriture de Daly et Miller (1975), adopté également par Cheng et al. (1999), qui est critiqué pour mesurer à la fois l'appréhension d'écriture, surtout dans une langue première, et le sentiment d'auto-effi- 
cacité relatif à l'écriture. Par conséquent, il est nécessaire de mettre au point une mesure de l'anxiété de production écrite qui ne confond pas la confiance en soi avec l'anxiété de production écrite. A cet effet, 67 étudiants diplômés de premier et de deuxième cycle venant de trois classes entières du département d'anglais ont créé un ensemble initial d'items de l'échelle d'anxiété de production écrite au moyen d'un questionnaire ouvert. L'ensemble d'items a ensuite été mis à l'essai par 56 étudiants de premier cycle. À l'étape finale, 421 étudiants d'anglais de première année de deuxième cycle, inscrits à des cours d'écriture en anglais dans différentes universités, ont complété les deux dernières questions. Les outils d'étude suivants ont été utilisés : les échelles de l'anxiété relative à l'utilisation de l'anglais et de l'anxiété en classe de Gardner (1985), le test d'attitude / d'appréhension d'écriture en anglais de Daly et Miller (1975), le questionnaire de blocage en écriture en anglais (Rose 1984), l'échelle de l'auto-efficacité d'écriture en anglais (Shell \& Murphy \& Bruning 1989), le rapport personnel de l'appréhension de communication à l'école supérieure (McCroskey 1970), l'échelle de l'anxiété de test (Sarason \& Ganzer 1962), l'échelle de l'anxiété de mathématiques (Betz 1978), l'échelle de motivation relative à l'écriture en anglais (Gardner 1985), l'échelle de l'anxiété d'état (Zuckerman 1960) et l'indice de la performance d'écriture en anglais (tâche consistant à écrire un essai en anglais dans un temps déterminé où on évalue l'organisation, le vocabulaire, l'utilisation de la langue et la mécanique).

Sur la base des résultats de la recherche a été développée une mesure à trois dimensions pour l'auto-évaluation de l'anxiété d'écriture dans une langue seconde / étrangère : Inventaire de l'anxiété de production écrite dans une langue seconde (SLWAI : Second Language Writing Anxiety Inventory), composé de trois sous-échelles : de l'anxiété somatique, de l'anxiété cognitive et du comportement d'évitement. La fiabilité et la validité de l'échelle et de ses trois sous-échelles ont été confirmées. L'échelle SLWAI a été jugée distincte de l'auto-efficacité de l'écriture dans une langue seconde, ce qui signifie que c'est un outil approprié pour analyser les relations entre l'anxiété de production écrite dans une langue seconde et la confiance en soi.

Le but de l'étude de Matsuda et Gobel (2004) est d'enquêter sur la relation entre l'anxiété langagière relative aux langues étrangères et l'anxiété de lecture. En dehors de cela, les auteurs veulent également analyser le rôle du sexe des apprenants, de leur compétence et de leur exposition à la culture cible afin d'établir les prédicteurs de la performance relative à l'utilisation d'une langue étrangère en classe. Ont participé à cette étude 252 étudiants de deuxième cycle, étudiant l'anglais à différents niveaux dans une université de Kyoto. Les principaux outils utilisés étaient les échelles FLCAS et FLRAS avec une question supplémentaire relative aux séjours de l'apprenant à l'étranger, ainsi qu'à ses notes de cours. 
Les résultats de la recherche confirment l'autonomie des concepts d'anxiété langagière et d'anxiété de lecture. En outre, les auteurs confirment leur hypothèse que les apprenants ayant des expériences de séjours à l'étranger déclarent une anxiété langagière inférieure ; ils ont de meilleures notes et leur confiance en soi en parlant anglais est renforcée. Cependant, le sexe n'a pas d'effet significatif sur les types d'anxiété en question ou sur leurs souscomposantes. Néanmoins, le sexe et la confiance en soi sont les éléments clés de la réussite parmi les étudiants de première année.

La compréhension orale est au centre de la recherche d'Elkhafaifi (2005). L'objet principal de l'étude est de vérifier la relation entre l'anxiété langagière et l'anxiété d'écoute ainsi que leur relation avec la réussite des apprenants. Les corrélats de ces types d'anxiété sont également étudiés. 233 étudiants suivant des cours de langue arabe à différents niveaux dans six universités américaines ont pris part à cette étude. Les outils utilisés étaient inclus dans un questionnaire qui comportait des questions de fond sur le sexe, l'année d'école, la durée de l'apprentissage de la langue arabe, le type de cours, d'autres langues étrangères et l'exposition à l'arabe parlé. Des échelles de l'anxiété d'écoute adaptées de l'échelle FLCAS, l'anxiété de lecture (FLRAS) de Saito et al. (1999) et l'échelle de l'anxiété langagière (FLCAS) ont également été utilisées. Les notes finales de cours et les notes de compréhension orale ont également été prises en considération.

L'anxiété langagière et l'anxiété d'écoute se révèlent des concepts distincts quoique liés entre eux. L'auteur émet l'hypothèse que des niveaux élevés d'anxiété langagière pourraient avoir un effet défavorable sur la performance et les compétences linguistiques de l'apprenant. Qui plus est, l'anxiété langagière et l'anxiété d'écoute montrent une relation négative significative avec les scores d'écoute et les notes de cours, notamment dans le cas des filles qui apparaissent nettement plus anxieuses. L'auteur conclut que les corrélations négatives significatives entre l'anxiété d'écoute, la compréhension d'écoute et la note finale du cours renforcent l'affirmation selon laquelle des niveaux d'anxiété plus élevés ont une incidence négative sur la performance générale, et pas seulement sur l'exécution des tâches liées à l'anxiété relative aux compétences.

Un outil pour évaluer l'anxiété d'écoute est proposé dans l'étude de Kim (2005). 312 participants à l'étude étaient des étudiants de deuxième cycle en sciences humaines, âgés de 19 à 26 ans. Les outils suivants ont été utilisés : l'échelle de l'anxiété d'écoute relative aux langues étrangères (FLLAS: Foreign Language Listening Anxiety Scale), l'Inventaire de l'Anxiété de Trait, le FLRAS, le FLCAS et l'échelle de désirabilité sociale de Crowne et Marlowe (1960). 
Les chercheurs constatent que le FLLAS est une échelle fiable et valide pour mesurer l'anxiété d'écoute. Sa corrélation avec l'anxiété de trait est faible, car l'anxiété d'écoute peut être distinguée de l'anxiété généralisée résultant du type de personnalité. Néanmoins, une forte corrélation est trouvée pour l'anxiété relative à l'apprentissage des langues étrangères en classe et le FLLAS, ce qui signifie que l'anxiété d'écoute est profondément liée à l'anxiété langagière. L'anxiété de lecture, cependant, est modérément corrélée avec l'anxiété d'écoute, parce qu'elles mesurent le même genre d'anxiété de compréhension. La corrélation négligeable de l'anxiété d'écoute avec la désirabilité sociale a montré que le FLLAS ne dépend pas de celle-ci, ce qui prouve que les résultats FLLAS ne sont pas déformés par ce facteur.

Les études décrites ci-dessus analysent le rôle des anxiétés relatives aux compétences dans l'apprentissage d'une langue étrangère. Elles prouvent à l'unanimité que la réussite dans l'apprentissage d'une langue est non seulement liée à l'anxiété langagière principalement due à l'aspect oral de l'utilisation d'une langue (l'anxiété d'expression orale), mais aussi à l'anxiété de lecture (Matsuda \& Gobel 2004 ; Saito et al. 1999), à l'anxiété d'écriture (Cheng et al. 1999) et à l'anxiété d'écoute (Elkhafaifi 2005 ; Kim 2005) qui peuvent être mesurées au moyen d'outils fiables. Dans le même temps, les anxiétés relatives aux compétences restent des phénomènes indépendants, ce qui montre que l'anxiété langagière est un concept plus général (Cheng et al. 1999), sans rapport avec la combinaison donnée d'une langue maternelle et d'une langue cible. D'autre part, l'anxiété de lecture varie en fonction de la langue cible (Saito et al. 1999). En outre, il faut ajouter que l'anxiété langagière est corrélée négativement avec les séjours à l'étranger et avec la réussite en langue (Matsuda \& Gobel 2004). Cet éventail d'études apporte encore des résultats contradictoires en ce qui concerne le rôle du sexe dans l'activation de l'anxiété langagière : soit le sexe est insignifiant (Matsuda \& Gobel 2004), soit les apprenants de sexe féminin sont plus anxieux (Elkhafaifi 2005).

Afin de décrire l'anxiété langagière de manière plus exhaustive, il est nécessaire d'analyser, en plus d'une étude précise des anxiétés relatives aux compétences linguistiques, son activation et les étapes du traitement de la langue.

\section{ANXIÉTÉ LANGAGIÈRE ET TRAITEMENT DE LA LANGUE}

Ce volet d'article consiste en une analyse de l'anxiété dans un environnement contrôlé au moyen d'une conception expérimentale permettant un plus grand degré de contrôle ou, comme dans les recherches précédentes, au moyen de projets descriptifs. Dans les sections qui suivent nous présentons 
la revue des recherches sur l'anxiété langagière relative aux trois étapes du traitement de la langue, à savoir l'input, le processing et l'output (Kalińska-Łuszczyńska 2015 : 56).

L'évaluation du cadre théorique de l'anxiété langagière de Horwitz et al. (1986) était le but principal de l'étude expérimentale réalisée par MacIntyre et Gardner (1989). Les chercheurs voulaient également analyser l'application du modèle de traitement cognitif proposé par Tobias (1986) en ce qui concerne l'activation de l'anxiété langagière à l'étape du traitement de la langue. 104 étudiants canadiens en introduction à la psychologie, locuteurs natifs d'anglais, ont participé à cette recherche. Plusieurs échelles d'anxiété ont été utilisées : les échelles d'anxiété en classe de français et d'anglais et les échelles d'utilisation du français, d'anxiété de trait, d'anxiété d'état, d'anxiété informatique et d'anxiété de test, ainsi que l'échelle d'anxiété en classe de mathématiques. En dehors de l'analyse de la sensibilité des sujets, ont également été utilisés un test de couples associés et un test de vocabulaire.

Les principales conclusions de cette étude confirment les conclusions de Horwitz et al. (1986) sur la relation négative entre la production linguistique (la tâche d'apprentissage des couples associés en rappel libre, des épreuves orales et des tests de vocabulaire écrits), l'appréhension de communication et l'anxiété d'évaluation sociale. D'autre part, l'anxiété de test semble être un problème plus général, indépendant de la classe de langue. En outre, l'anxiété se révèle avoir une influence relativement stable sur le travail avec la langue à toutes les étapes du traitement de celle-ci, de l'input à l'output. Les corrélations négatives significatives entre une mesure donnée d'anxiété langagière (anxiété de la classe de français) et la performance relative à une tâche d'apprentissage de vocabulaire ont conduit à la conclusion que l'anxiété langagière est la cause d'une mauvaise performance en langue étrangère à cause d'un niveau croissant d'anxiété d'état, ce qui, à son tour, conduit à l'anxiété situationnelle, c'est-à-dire à l'anxiété langagière.

Une autre étude expérimentale reproduisant les conclusions ci-dessus, également en référence à la langue maternelle, a été réalisée par MacIntyre et Gardner (1991a) avec 95 étudiants en première année de psychologie apprenant le français. Les outils utilisés comprennent les tests Digit Span (test de mémoire des chiffres) et Thing Category (test de vocabulaire), ainsi que 23 échelles mesurant différents types d'anxiété, comme l'anxiété relative à l'apprentissage des langues étrangères (Anxiété de classe et d'utilisation du français de MacIntyre \& Gardner 1988) ou l'anxiété en mathématiques (des mêmes auteurs) et la peur de l'évaluation négative (Watson \& Friend 1969), avec un anxiomètre, un dessin ressemblant à un thermomètre avec une échelle de 10 points sur laquelle les participants indiquent leur niveau d'anxiété. 
Les résultats obtenus dans cette recherche confirment les conclusions précédentes, à savoir que l'anxiété langagière est distincte des autres formes d'anxiété. En outre, différentes dimensions de l'anxiété sont établies, avec les principaux aspects suivants : anxiété générale et communicative, anxiété d'état et anxiété de trait, anxiété de nouveautés et anxiété liée aux situations d'utilisation du français (langue étrangère). L'anxiété de langue est en corrélation avec les mesures de performance, conduisant à l'observation que l'anxiété a un impact négatif sur les étapes d'input et d'output du traitement de la langue. En ce qui concerne la spécificité linguistique, les tâches en langue étrangère se sont avérées induire plus d'anxiété que celles en langue maternelle.

L'objet principal d'une autre étude expérimentale de MacIntyre et Gardner (1994a) était d'analyser l'éveil de l'anxiété causée par l'utilisation d'une caméra vidéo à trois étapes de l'apprentissage, en référence à l'apprentissage et à la production du vocabulaire. 72 étudiants fréquentant des cours de français de première année ont participé à l'étude. Ils ont été répartis au hasard en quatre groupes. Dans les trois groupes expérimentaux, une caméra vidéo a été introduite avant les trois étapes du traitement de la langue : l'input, le traitement et l'output, avec l'intention de susciter l'anxiété à ces stades. Pendant l'étude, les participants ont été tenus d'apprendre 19 paires de substantifs français-anglais. Plusieurs techniques ont été utilisées, notamment l'anxiomètre, des tâches de performance (cf. MacIntyre \& Gardner 1994b), un test de mémoire des chiffres, un test de catégories du vocabulaire, une auto-description (description de soi pendant une minute dans deux langues), et une tâche de rappel du vocabulaire (réponses orales à des questions en français).

Les résultats de l'étude suggèrent que les niveaux d'anxiété plus élevés apparaissent immédiatement après l'introduction de la caméra. Aussi les étudiants ayant les scores les plus élevés dans les mesures de l'anxiété ont-ils les pires résultats à ces tâches. La performance des groupes de contrôle permet de formuler l'observation qu'une simple tâche communicative est plus anxiogène qu'une tâche d'apprentissage. L'anxiété suscitée par la caméra vidéo nuit à la performance des apprenants, notamment aux étapes du traitement et de l'output.

Une autre étude de MacIntyre et Gardner (1994b) tentait d'examiner l'anxiété dans les processus cognitifs spécifiques impliqués dans le traitement de la langue au moyen d'un modèle d'apprentissage en trois étapes consécutives : input, traitement et output. Les participants étaient 97 étudiants de première année apprenant le français comme langue seconde dans une université canadienne anglophone. Aux fins de la recherche, les auteurs 
ont développé trois mesures de l'anxiété dont le rôle était d'estimer l'anxiété aux étapes du traitement de la langue : l'anxiété d'input, l'anxiété de traitement et l'anxiété d'output. En outre, des mesures de l'anxiété ont été utilisées pour évaluer la validité de ces nouvelles échelles : l'anxiété en classe de français (Gardner 1985), l'anxiété de l'utilisation du français (MacIntyre \& Gardner 1988) et le FLCAS. D'autres techniques ont utilisé des mesures de la compétence (les notes de cours, la réussite en français) et des tâches représentant les étapes respectives (Word Span - mémorisation de chaînes de mots, Digit Span - mémorisation d'une série de chiffres, T-scope - reconnaissance de mots, traduction de paragraphes, apprentissage par couples associés ou mots correspondants, Thing Category - production de vocabulaire, texte à trous et auto-description).

Les résultats principaux prouvent que les niveaux d'anxiété relatifs à ces étapes sont fortement corrélés avec les tâches spécifiques à une étape donnée, ce qui suggère que les effets de l'anxiété langagière ont tendance à être à la fois omniprésents et subtils. En outre, l'anxiété langagière est en corrélation avec les mesures de la performance en langue seconde, mais pas nécessairement en langue première, avec des évaluations générales de compétence (tests de compétence et notes normalisées) liées négativement à l'anxiété. Les auteurs supposent que les apprenants anxieux ont plus de mal à montrer leurs connaissances, mais, en même temps, ils ont une base de connaissances plus petite en langue seconde.

La validation psychométrique des échelles mesurant l'anxiété langagière aux étapes d'input, de traitement et d'output, ainsi que la vérification de l'exactitude de ces échelles dans la mesure et la description du modèle en trois étapes de MacIntyre et Gardner (1994b) étaient l'objet principal de la recherche effectuée par Onwuegbuzie et al. (2000). 258 étudiants inscrits à des cours d'espagnol, de français et d'allemand à des niveaux débutant, intermédiaire et avancé dans une université aux États-Unis ont été analysés à l'aide des échelles de l'anxiété d'input, de traitement et d'output (MacIntyre \& Gardner 1994b). D'autres échelles ont été utilisées : l'échelle de l'anxiété de français en classe (Gardner 1985), celle de l'anxiété d'utilisation du français (MacIntyre \& Gardner 1988) et l'échelle FLCAS.

Ces trois échelles mesurant l'anxiété à trois étapes du traitement de la langue, analysées séparément, s'avèrent posséder des caractéristiques psychométriques adéquates. Cependant, l'analyse des facteurs de confirmation ne corrobore pas l'unidimensionnalité du concept d'anxiété langagière, tout en confirmant cependant le modèle en trois étapes. En outre, les apprenants déclarent éprouver des niveaux d'anxiété d'output des plus élevés. Enfin, l'anxiété d'input s'avère être liée à l'anxiété langagière de la manière la plus forte qui soit. 
Bailey, Onwuegbuzie et Daley (2000) cherchaient à analyser une combinaison de variables concernant l'anxiété langagière mesurée aux étapes du traitement de la langue et comme concept séparé (FLCAS). Les prédicteurs de l'étude étaient sept mesures de la perception de soi (l'estime de soi perçue, la capacité intellectuelle perçue, la compétence scolaire perçue, le nombre de cours de langues étrangères suivis à l'école secondaire, la compétence de l'emploi perçue, la créativité perçue et l'attente de la réussite en langues étrangères), trois mesures de l'interdépendance sociale (l'esprit de coopération, la compétitivité et l'individualisme) et quatre caractéristiques démographiques (le sexe, l'âge, le nombre de pays étrangers visités et la réussite scolaire mesurée par la moyenne pondérée cumulative). Y ont participé 205 étudiants fréquentant des cours d'espagnol, de français et d'allemand, aux niveaux débutant, intermédiaire et avancé dans une université aux États-Unis. Les outils utilisés ont été notamment les suivants : l'échelle de l'anxiété d'input, l'échelle de l'anxiété de traitement, l'échelle de l'anxiété d'output (MacIntyre \& Gardner 1994b), le profil d'autoperception pour les étudiants de collège (Neemann \& Harter 1986), l'échelle d'interdépendance sociale (Johnson \& Norem-Hebeisen 1979) et un formulaire démographique avec des informations sur l'âge, le sexe, la langue maternelle, les pays visités et l'attente de réussite dans le cours de langue suivi.

Les principales conclusions de l'étude montrent que les prédicteurs significatifs de l'anxiété langagière sont l'âge, l'expérience préalable à l'école secondaire en langues étrangères, la moyenne générale de cours attendue, la compétence scolaire perçue et l'estime de soi perçue ainsi que la capacité intellectuelle perçue et la compétence de l'emploi perçue. Les résultats suggèrent également que les échelles de MacIntyre \& de Gardner (1994b) peuvent être utilisées comme alternative à l'échelle FLCAS. Un autre aspect souligne la connexion importante de l'anxiété de traitement avec l'anxiété langagière. L'anxiété et la perception de soi s'avèrent aussi fortement corrélées avec l'attente de la réussite dans l'apprentissage d'une langue étrangère, ce qui confirme la théorie du filtre affectif de Krashen (1980) et la déclaration de MacIntyre et Gardner (1991b : 110), selon laquelle l'anxiété langagière relative aux langues étrangères résulte $d$ ' " attentes négatives qui conduisent à des sentiments d'inquiétude et à une émotivité ". L'âge est un puissant prédicteur de l'anxiété langagière : les apprenants plus âgés ont des niveaux plus élevés d'anxiété d'input, de traitement et d'output. Les auteurs attribuent cette constatation à plusieurs facteurs. Tout d'abord, on remarque une baisse liée à l'âge de la capacité à maîtriser les subtilités de la langue et de la capacité à parler une langue étrangère sans accent. Deuxièmement, les personnes plus âgées hésitent à prononcer, à traduire ou à écrire des mots dans une langue étrangère qu'elles n'ont pas totalement maîtrisée. Elles ont éga- 
lement tendance à faire plus d'erreurs d'omission que de commission, et mettent l'accent sur la précision plus souvent que les jeunes adultes. Une autre constatation importante est que les apprenants sans expérience préalable relative aux langues étrangères ont des niveaux plus élevés d'anxiété $d$ 'input, de traitement et d'output. Cela souligne l'importance d'encourager les élèves à apprendre une langue étrangère à l'école secondaire ou même à l'école primaire.

L'étude de Frantzen et de Magnan (2005) se concentrait sur l'analyse des différences dans les réactions d'anxiété des vrais et faux débutants, comme les différences dans l'anxiété aux étapes du traitement de la langue et les différences relatives aux notes finales attendues, aux projets des apprenants sur la poursuite de leur apprentissage de la langue étrangère ainsi qu'aux sources de confort. L'étude comptait 490 participants, étudiants en premier semestre dans une université américaine fréquentant des cours de français et d'espagnol (de vrais débutants sans expérience de la langue et de faux débutants ayant eu une expérience de la langue à l'école secondaire ou dans le premier cycle de l'enseignement supérieur). Deux outils ont été utilisés dans l'étude : un questionnaire et un entretien. Le premier outil était l'échelle FLCAS et l'échelle d'anxiété de MacIntyre et Gardner (1989 ; 1994a) qui mesurait l'anxiété connue aux trois étapes du traitement de la langue : anxiété $\mathrm{d}^{\prime}$ input, de traitement et d'output. Les entretiens individuels portaient sur la description des réactions de chaque apprenant relatives à l'anxiété.

Les résultats principaux obtenus dans cette étude conduisent à la conclusion que les étudiants débutants sont nettement plus anxieux que les faux débutants, même à la fin du semestre. Les mêmes résultats sont obtenus en référence à l'anxiété aux étapes de traitement et d'output, en dehors de l'étape d'input, où les deux groupes d'étudiants sont également anxieux, ce qui signifie que les réactions d'anxiété des apprenants ne sont pas différentes quand ceux-ci répètent de nouvelles informations et les intègrent au savoir déjà acquis. De même, les vrais débutants s'attendent à des notes inférieures et les obtiennent. En outre, plus de vrais débutants que de faux débutants envisagent de continuer à apprendre une langue étrangère donnée. Selon les participants, l'enseignant et une communauté de classe bienveillante sont les outils les plus importants permettant de réduire l'anxiété.

L'anxiété langagière des apprenants débutants adultes est également analysée dans une étude réalisée par Turula (2006). Plus précisément, sa recherche se concentre généralement sur la relation entre l'anxiété langagière et la dynamique de classe, ainsi que sur l'anxiété connue à l'étape du traitement de la langue. 45 étudiants débutants adultes ont pris part à l'étude, inscrits dans quatre groupes avec différents enseignants et différentes prati- 
ques pédagogiques. Les techniques utilisées aux fins de l'étude ont été un questionnaire utilisant, entre autres, les mesures de l'anxiété aux étapes du traitement de la langue (MacIntyre \& Gardner 1989) et les opinions des apprenants sur les enseignants, groupes et salles de classe parfaits, ainsi que les observations réalisées en classe.

Les évaluations de l'anxiété langagière montrent que l'anxiété identifiée comme la plus élevée par la plupart des participants est l'anxiété d'input. Les étudiants se plaignent de l'anglais parlé trop vite et d'une manière confuse. Ils se plaignent également des notes confuses, des classes mal organisées et de leur incapacité à se rappeler les mots et à réussir les tests. Ils demandent fréquemment de répéter ou d'expliquer les questions peu claires et ils se plaignent de problèmes de concentration. L'auteur suppose que les niveaux d'anxiété langagière dépendent de l'écart entre l'enseignant idéal et la réalité de la classe. Cette anxiété reste également en corrélation négative avec l'autonomie de l'apprenant et sa capacité à contrôler son propre progrès et à organiser son temps et les sujets d'apprentissage. Une autre importante source d'anxiété est l'inquiétude de l'apprenant à propos de son échec potentiel et une disposition des sièges entravant les tentatives de communication.

L'anxiété à l'étape du traitement de la langue, à savoir l'anxiété d'input, de traitement et d'output, possède des caractéristiques similaires à l'anxiété langagière : elle est corrélée négativement avec la réussite (Frantzen \& Magnan 2005 ; MacIntyre \& Gardner 1989 ; 1991a, 1994a ; 1994b) et avec l'expérience de la langue (Bailey et al. 2000). Elle est également liée aux causes principales de l'anxiété langagière, comme l'appréhension de communication et l'anxiété de l'évaluation sociale (MacIntyre \& Gardner 1989 ; Turula 2006). Parmi d'autres corrélats de l'anxiété à l'étape du traitement de la langue, on trouve : la moyenne générale de cours attendue, la compétence scolaire perçue et l'estime de soi perçue, ainsi que la capacité intellectuelle perçue et la compétence de l'emploi perçue (Bailey et al. 2000). L'anxiété d'output semble être la plus aiguë chez les apprenants de langue (MacIntyre \& Gardner 1994a ; 1994b ; Onwuegbuzie et al. 2000). Néanmoins, d'autres types d'anxiété, comme l'anxiété de traitement et d'input, ont également un rôle important dans le traitement de la langue. L'anxiété d'input est liée à l'anxiété de langue de la manière la plus forte (Onwuegbuzie et al. 2000), indépendamment de l'expérience du langage (Turula 2006), tandis que l'anxiété de traitement joue également un rôle important (Frantzen \& Magnan 2005). Les échelles de mesure de l'anxiété aux étapes du traitement de la langue possèdent des caractéristiques psychométriques adéquates et confirment le modèle de l'anxiété en trois étapes (Onwuegbuzie et al. 2000). 


\section{CONCLUSION}

Ces études fournissent une image plus claire de l'anxiété langagière, en analysant en même temps certains de ses corrélats. Pourtant, une vision plus complète des corrélations de l'anxiété langagière doit être prise en compte.

La relation entre le domaine cognitif et affectif montre que l'un ne peut pas fonctionner correctement sans l'autre. Les domaines affectif et cognitif sont les deux faces de la même médaille. Ainsi, l'analyse de tous ces aspects est très importante. Sinon, une mauvaise polarisation du domaine affectif rendrait le système d'éducation futile.

Incontestablement, les émotions, notamment l'anxiété, diffusent des informations au sujet de son état interne de tension. Cet état de conflit interne a un effet sur la réussite des étudiants. Par conséquent, la réussite des étudiants anxieux devient considérablement moins probable.

Parler dans une langue étrangère implique des émotions qui peuvent être intenses, négatives et positives. Comme c'est par la prise de parole que les enseignants sont plus en mesure d'apprécier les progrès de leurs élèves, les tâches orales continueront d'être une partie régulière des classes de langue étrangère.

Sensibiliser les enseignants de langues étrangères à l'égard des émotions que leurs élèves peuvent éprouver et aider les élèves à discuter de leurs sentiments à l'égard de la prise de parole en langue étrangère, publiquement et en privé, devraient être des éléments importants de chaque classe de langue. Cela conduira, comme on peut l'espérer, à une amélioration des compétences de prise de parole et des comportements des étudiants relatifs à l'apprentissage de la langue étrangère.

\section{RÉFÉRENCES}

Argaman, O. / Abu-Rabia, S. (2002). The influence of language anxiety on English reading and writing tasks among native Hebrew speakers. Language, Culture and Curriculum, 15(2), pp. 143-160.

Bailey, P. / Onwuegbuzie, A. J. / Daley, C. E. (2000). Correlates of anxiety at three stages of the foreign language learning process. Journal of Language and Social Psychology, 19, pp. 474-490.

Betz, N. (1978). Prevalence, distribution, and correlates of math anxiety in college students. Journal of Counseling Psychology, 25/5, pp. 441-48.

Cheng, Y. S. (2004). A measure of second language writing anxiety: Scale development and preliminary validation. Journal of Second language Writing, 13, pp. 313-335.

Cheng, Y. S. / Horwitz, E. K. / Schallert, D. L. (1999). Language writing anxiety: Differentiating writing and speaking components. Language Learning, 49, pp. 417-446.

Crowne, D. P. / Marlowe, D. A. (1960). A new scale of social desirability independent of psychopathology. Journal of Consulting Psychology, 24, pp. 349-354. 
Daly, J. A. / Miller, M. D. (1975). The empirical development of an instrument of writing apprehension. Research in the Teaching of English, 9, pp. 242-249.

Elkhafaifi, H. (2005). Listening comprehension and anxiety in the Arabic language classroom. The Modern Language Journal, 89, pp. 206-220.

Frantzen, D. / Magnan, S. (2005). Anxiety and the true beginner-false beginner dynamic in beginning French and Spanish classes. Foreign Language Annals, 38(2), pp. 171-190.

Gardner, R. C. (1985). Social psychology and second language learning: the role of attitudes and motivation. London: Edward Arnold.

Horwitz, E. K. / Horwitz, M. B. / Cope, J. A. (1986). Foreign language classroom anxiety. The Modern Language Journal, 70(2), pp. 125-132.

Johnson, D. W. / Norem-Hebeisen, A. A. (1979). A measure of cooperative, competitive, and individualistic attitudes. The Journal of Social Psychology, 109, pp. 253-261.

Kalińska-Łuszczyńska, S. (2015). La communication médiatisée par ordinateur et la réduction de l'anxiété langagière chez les étudiants de langue étrangère. In: B. Kędzia-Klebeko / S. Kalińska-Łuszczyńska (éd.), Contextes médiatiques et matériaux didactiques dans l'enseignement des langues étrangères (pp. 49-73). Szczecin : Uniwersytet Szczeciński.

Kim, E. J. (2005). The effect of decreased safety behaviors on anxiety and negative thoughts in social phobics. Journal of Anxiety Disorders, 19, pp. 69-86.

Krashen, S. (1980). The theoretical and practical relevance of simple codes in second language acquisition. In: R. Scarcella / S. Krashen (éd.). Research in second language acquisition (pp. 7-8). Rowley, MA: Newbury House.

MacIntyre, P. D. / Gardner, R. C. (1988). The measurement of anxiety and applications to second language learning: An annotated bibliography. Research Bulletin, 672, pp. 1-39.

MacIntyre, P. D. / Gardner, R. C. (1989). Anxiety and second language learning: toward a theoretical clarification. Language Learning, 32, pp. 251-275.

MacIntyre, P. D. / Gardner, R. C. (1991a). Language anxiety: Its relation to other anxieties and to processing in native and second languages. Language Learning, 41(4), pp. 513-534.

MacIntyre, P. D. / Gardner, R. C. (1991b). Methods and results in the study of anxiety in language learning: A review of the literature. Language Learning, 41, pp. 85-117.

MacIntyre, P. D. / Gardner, R. C. (1994a). The effects of induced anxiety on cognitive processing in computerized vocabulary learning. Studies in Second Language Acquisition, 16, pp. 1-17.

MacIntyre, P. D. / Gardner, R. C. (1994b). The subtle effects of language anxiety on cognitive processing in the second language. Language Learning, 44, pp. 283-305.

Matsuda, S. / Gobel, P. (2004). Anxiety and predictors of performance in the foreign language classroom. System, 32, pp. 21-36.

McCroskey, J. C. (1970). Measures of communication-bound anxiety. Speech Monographs, 37, pp. 269-277.

Neemann, J. / Harter, S. (1986). Manual for the self-perception profile for college students. Colorado: University of Denver.

Onwuegbuzie, A. J. / Slate, J. / Paterson, F. / Watson, M. / Schwartz, R. (2000). Factors associated with underachievement in educational research courses. Research in the Schools, 7, pp. 53-65.

Rose, M. (1984). Writer's block: The cognitive dimension. Carbondale: Southern Illinois University Press.

Saito, Y. / Horwitz, E. K. / Garza, T. J. (1999). Foreign language reading anxiety. The Modern Language Journal, 83, pp. 202-218. 
Sarason, I. / Ganzer, V. J. (1962). Anxiety, reinforcement, and experimental instructions in a free verbal situation. Journal of Abnormal and Social Psychology, 65, pp. 300-307.

Shell, D. F. / Murphy, C. C. / Bruning, R. H. (1989). Self-efficacy and outcome expectancy mechanisms in reading and writing achievement. Journal of Educational Psychology, 81, pp. 91-100.

Tobias, S. (1986). Anxiety and cognitive processing of instruction. In: R. Schwarzer (éd.), Self-related cognition in anxiety and motivation (pp. 35-54). Hillsdale, NJ: Erlbaum.

Turula, A. (2006). Language anxiety and classroom dynamics. Bielsko-Biała: Wydawnictwo Naukowe ATH.

Watson, D. / Friend, R. (1969). Measurement of social-evaluative anxiety. Journal of Consulting and Clinical Psychology, 33, pp. 448-457.

Zuckerman, M. (1960). The development of an affect adjective check list for the measurement of anxiety. Journal of Consulting Psychology, 24, pp. 457-462.

Received: 17.04.2016; revised: 4.04.2017 
